\title{
THE CYCLIC PERIOD VARIABILITY IN SW CYGNI
}

\author{
ROBERT C. BERRINGTON AND DOUGLAS S. HALL \\ Dyer Observatory, Vanderbilt University, Nashville, Tennessee 37235 \\ Electronic mail: hallxxds@ctrvax.vanderbilt.edu \\ Received 1993 August 17; revised 1994 January 6
}

\begin{abstract}
Timings of primary eclipse up through 1992 are collected, plus a few overlooked in earlier studies. Analysis shows that, after decreasing until $\approx 1919$ and increasing since then, the period has now started decreasing again, with the inflection point of the $\mathrm{O}-\mathrm{C}$ curve being $\approx 1972$. The shortest period was $4 \div 5727$, the longest 4.5732 , with a full cycle length of 96 yr. Explanation by the time-delay effect, due to orbital motion around a third body, would require an unseen black hole companion of $\geqslant 10 \mathscr{U}_{\odot}$. A magnetic cycle operating in the $\mathrm{K}$-type subgiant, an active dynamo star because of its convection and rapid rotation, is the best explanation.
\end{abstract}

\section{INTRODUCTION}

SW Cygni was discovered as a variable star in 1898 and is important even today as an Algol-type eclipsing binary which is not extremely faint $\left(V=9 .{ }^{\mathrm{M}} 3\right.$ at maximum), undergoes well-defined complete eclipses $(d=0 \stackrel{d}{d} 11)$, and has a dramatically variable orbital period (of approximately 4.573 ) defined by eclipse timings now covering almost a full century. A subgiant with color indices implying a spectral type of early $\mathrm{K}$ is filling its Roche lobe and transferring mass onto the more luminous $\mathrm{A} 2 \mathrm{~V}$ companion star.

The period was noted as variable long ago, first by Blazko (1925). Since then many numerical schemes have been employed to represent the shape of the $\mathrm{O}-\mathrm{C}$ curve: a single parabola, a cubic, a sinusoid, straight-line segments, parabolic segments, a Keplerian orbit, and various combinations of those. Physical mechanisms invoked to account for the period variability have included orbital motion around a third body, apsidal motion, and mass transfer. The most recent three papers to concentrate on the period variability are those of Frieboes-Conde \& Herczeg (1973), Hall et al. (1979), and Chaubey (1980).

The most important papers to deal with the eclipse light curve, its photometric complications, and its solution are those of Walter (1971), Hall \& Garrison (1972), Hall et al. (1979), Olson (1982), and Wilson \& Mukherjee (1988). The last of these concluded that SW Cyg is in "double contact," by which they mean that the K-type subgiant fills its Roche lobe while the A2 V star is rotating so much faster than synchronously as to be at its centrifugal breakup limit.

Despite work to date, the period variability in SW Cyg is frankly not satisfactorily understood. The period was about $4 \mathrm{~d} 57294$ during the decade before its discovery (Pickering 1899), 4.5729 around the epoch of its discovery, decreased further to about 4.5727 as of about 1919 , and then increased thereafter, being about 4.5732 as of 1977 , the year of the last eclipse timings discussed by Hall et al. (1979) and by Chaubey (1980) (note that these are huge period changes, with a full range of $\left.\triangle P / P=10^{-4}\right)$. Such behavior simply cannot be accounted for (in a physically consistent way) by apsidal motion, orbital motion around a third body, mass transfer, or mass loss. The arguments are given in detail by
Hall (1990), although SW Cyg was not mentioned specifically in that review. Briefly, the absence of orbital eccentricity rules out apsidal motion, a third body of unreasonably large mass would be required, most modes of mass transfer and/or mass loss produce only monotonic period changes, and the Biermann-Hall model (which can accomodate period changes of both sign in the same binary system) has been shown not to be correct.

Work since the most recent period studies on SW Cyg (Hall et al. 1979; Chaubey 1980) indicates that period variability of this sort is best understood as a manifestation of a magnetic cycle operating in the $\mathrm{K}$-type subgiant, which is a convective star in a state of rapid rotation and hence expected to be experiencing strong dynamo action. For background on the occurrence of magnetic cycles in stars of various different types, see Hall (1990). For the relation between rotation, convection, and dynamo action, see Hall (1991a). For the connection between magnetic cycles and changes in orbital period, when the active star happens to be in a binary, see Hall (1991b) and Applegate (1992). For the specific case of the active star filling its Roche lobe and hence being responsible for mass loss and mass transfer as well, see Hall (1989) and Tout \& Hall (1991).

In this paper we collect the most recent times of minimum, up through July 1992, plus a few others which earlier investigators had missed, and analyze them to show that the period has started decreasing again. This finding is, in a sense, confirmation of an implicit prediction of the magnetic cycle interpretation. With epochs of decreasing, increasing, and again decreasing period now in hand, we can determine the length of the hypothesized cycle relatively precisely.

\section{AVAILABLE TIMES OF MINIMUM, THROUGH 1992}

All independent timings of primary minimum which we have been able to locate are listed in Table 1 (this table is presented in its complete form in the ApJ/AJ CD-ROM Series, Vol. 2, 1994) and plotted in Fig. 1. Cycle numbers and $\mathrm{O}-\mathrm{C}$ residuals for each have been computed with the ephemeris 
TABLE 1. Times of primary minimum for SW Cygni.*

\begin{tabular}{|c|c|c|c|c|c|}
\hline $\begin{array}{l}\text { cycle } \\
\text { number } \\
\end{array}$ & $\begin{array}{l}\begin{array}{l}0-C \\
\text { (days) }\end{array} \\
\end{array}$ & $\begin{array}{l}\text { O-C' } \\
\text { (days) }\end{array}$ & $\begin{array}{l}\text { JD (he1.) } \\
2400000+\end{array}$ & $\begin{array}{l}\text { error } \\
\text { (days) }\end{array}$ & $\begin{array}{l}\text { ref. } \\
\text { no. }\end{array}$ \\
\hline-4391 & +0.6262 & -0.0090 & 13081.322 & 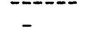 & 20 \\
\hline-4309 & +0.6435 & +0.0142 & 14456.324 & 1 & 1 \\
\hline-4069 & +0.6083 & -0.0037 & 14553.805 & 1 & 1 \\
\hline-4029 & +0.6159 & +0.0068 & 14736.732 & 1 & 1 \\
\hline-4019 & +0.6021 & -0.0063 & 14782.448 & 3 & 1 \\
\hline-4016 & +0.6271 & +0.0189 & 14796.192 & 1 & 1 \\
\hline-4014 & +0.6072 & -0.0008 & 14805.318 & 3 & 1 \\
\hline-4013 & +0.6072 & -0.0007 & 14809.891 & 1 & 1 \\
\hline-4012 & +0.6042 & -0.0037 & 14814.461 & 1 & 1 \\
\hline-4009 & +0.6063 & -0.0014 & 14828.182 & 1 & 1 \\
\hline-4008 & +0.6033 & -0.0043 & 14832.752 & 3 & 1 \\
\hline-4007 & +0.6053 & -0.0022 & 14837.327 & 3 & 1 \\
\hline-4005 & +0.6023 & -0.0051 & 14846.470 & 1 & 1 \\
\hline-4005 & +0.6063 & -0.0011 & 14846.474 & 3 & 1 \\
\hline-4005 & +0.6103 & +0.0029 & 14846.478 & 1 & 1 \\
\hline-4003 & +0.6104 & +0.0032 & 14855.624 & 1 & 1 \\
\hline-4002 & +0.6094 & +0.0023 & 14860.196 & 1 & 1 \\
\hline-4001 & +0.6044 & -0.0027 & 14864.764 & 1 & 1 \\
\hline-4000 & +0.6134 & +0.0064 & 14869.346 & 1 & 1 \\
\hline-4000 & +0.6154 & +0.0084 & 14869.348 & 1 & 1 \\
\hline-3998 & +0.6064 & -0.0005 & 14878.485 & 1 & 1 \\
\hline-3993 & +0.6105 & +0.0040 & 14901.354 & 1 & 1 \\
\hline-3989 & +0.5916 & -0.0146 & 14919.627 & 1 & 1 \\
\hline-3989 & +0.5986 & -0.0076 & 14919.634 & 1 & 1 \\
\hline-3986 & +0.6016 & -0.0044 & 14933.356 & 3 & 1 \\
\hline-3986 & +0.6056 & -0.0004 & 14933.360 & 1 & 1 \\
\hline-3984 & +0.6037 & -0.0021 & 14942.504 & 1 & 1 \\
\hline-3983 & +0.6117 & +0.0059 & 14947.085 & 1 & 1 \\
\hline-3982 & +0.6037 & -0.0020 & 14951.650 & 1 & 1 \\
\hline-3979 & +0.6147 & +0.0092 & 14965.380 & 1 & 1 \\
\hline-3975 & +0.6038 & -0.0014 & 14983.661 & 1 & 1 \\
\hline-3968 & +0.6069 & +0.0022 & 15015.675 & 1 & 1 \\
\hline-3965 & +0.6050 & +0.0005 & 15029.392 & 1 & 1 \\
\hline-3961 & +0.5980 & -0.0062 & 15047.677 & 1 & 1 \\
\hline-3949 & +0.6142 & +0.0109 & 15102.569 & 1 & 1 \\
\hline-3942 & +0.6073 & +0.0045 & 15134.573 & 1 & 1 \\
\hline-3937 & +0.6094 & +0.0069 & 15157.440 & 1 & 1 \\
\hline-3935 & +0.5974 & -0.0049 & 15166.574 & 1 & 1 \\
\hline-3934 & +0.5994 & -0.0028 & 15171.149 & 1 & 1 \\
\hline-3928 & +0.6125 & +0.0107 & 15198.600 & 1 & 1 \\
\hline-3923 & +0.5916 & -0.0098 & 15221.444 & 1 & 1 \\
\hline-3919 & +0.5907 & -0.0105 & 15239.735 & 1 & 1 \\
\hline-3916 & +0.5967 & -0.0042 & 15253.460 & 2 & 1 \\
\hline-3911 & +0.5908 & -0.0098 & 15276.319 & 1 & 1 \\
\hline-3906 & +0.6109 & +0.0107 & 15299.204 & 1 & 1 \\
\hline-3888 & +0.5982 & -0.0007 & 15381.505 & - & 20 \\
\hline-3886 & +0.6002 & +0.0014 & 15390.653 & 1 & 1 \\
\hline-3883 & +0.5973 & -0.0013 & 15404.369 & 1 & 1 \\
\hline
\end{tabular}

Note to TABLE 1

*Table 1 is presented in its complete form in the ApJ/AJ CD-ROM Series, volume 2,1994 . The first page of this table is presented here for guidance regarding its form and content.

$$
\begin{aligned}
C(\text { pri.min. })= & J D(\text { hel. }) 2433160.669 \\
& +4 \cdot 5729841 E
\end{aligned}
$$

which had been used by Frieboes-Conde \& Herczeg (1973, Eq. 8-I). Any uncertainty or error specified in the original source has been included in column 5 . In one case the original source published the timing only to the nearest 0.01 , in another case to the nearest hour, so for these we estimated the uncertainty ourselves. The 137 individual eclipse timings tabulated by Slovochotova (1954) are included in Table 1 but credited to Slovochotova herself, to keep the bibliography in our paper from being overly long. The weights she had assigned are listed in column 5 , in lieu of error estimates. Two times omitted by Slovochotova but included in our Table 1 were the ones at $E=-4391$ (Parenago 1931, Table 4) and at $E=-3888$ (Parenago 1931, table 3). Also, we corrected the 10 -cycle error in the time now appearing (correctly) at $E$ $=-2109$. Reference number 11 refers to various Bulletins of the B.B.S.A.G., too numerous to list separately. Similarly,

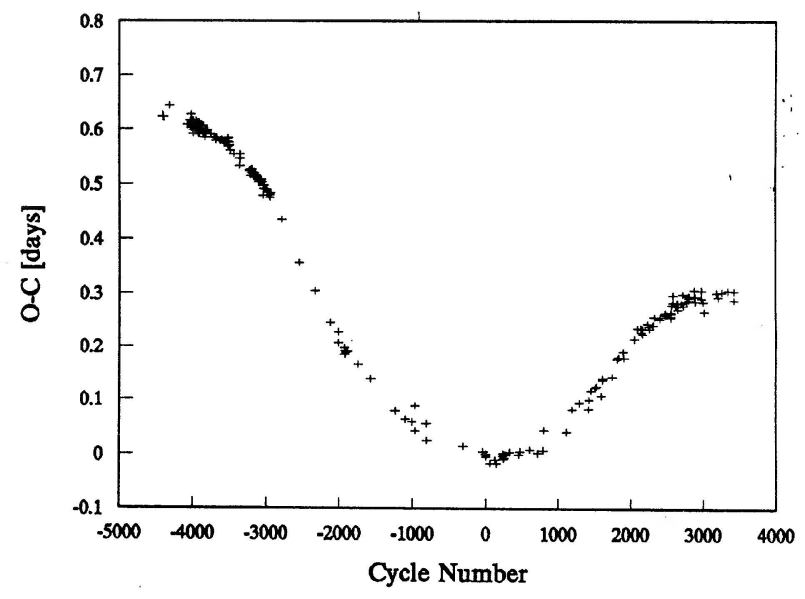

Fig. 1. The O-C curve for times of primary minimum of SW Cygni, computed for every timing in table 1 with the ephemeris in Eq. (1). After decreasing until $E=-2456(\approx 1919)$ and increasing since then, the period has now started decreasing again, with the inflection point of the $\mathrm{O}-\mathrm{C}$ curve being at $E=+1824(\approx 1972)$.

reference number 17 refers to the B.A.A.V.S.S. Circulars, numbers 60,61 , and 64 .

The 137 timings listed by Slovochotova (1954), and the two she had omitted, include ones determined both photographically and visually, with the latter including both visual estimates and observations with a visual photometer. All of the ones after Slovochotova are based on visual estimates, except for one which was spectroscopic (Ref. no. 18), four photographic (Ref. nos. 2 and 16), and 18 photoelectric (Ref. nos. $6,7,8,9,10,12,13)$.

Table 1 omits several published timings which are second-hand citations or means of individual timings which we do include. These are the 10 in Schneller (1963), the 3 in Lange (1959a) which reappear as a timing of a mean light curve in Lange (1959b), the other 4 in Lange (1959b), the 8 in Mallama (1980), and the single times in Wood \& Forbes (1963), in Rafert (1982), and in Isles (1988).

\section{FITTING THE O-C CURVE}

We used least squares to fit the O-C curve with a series of straight-line segments. The number of segments and the points included in each were decided upon by trial and error, to optimize the goodness of the overall fit. The results are given in Table 2 . The first column is a letter designating the segment. The second is the number of eclipse timings included in each fit. The third is the slope expressed as a difference from the period in Eq. (1). The fourth is the standard deviation of each fit. The last two are the cycle number and $\mathrm{O}-\mathrm{C}$ value of the intersection point between adjacent segments.

In segments $\mathrm{H}$ and $\mathrm{I}$, we used only the photoelectric timings within the time intervals included, three in both cases. In segment $\mathrm{G}$, which was very sparsely and very poorly covered, we gave the one photoelectric timing at $E=+1190$ infinite weight, gave the other four equal weight, and solved for the slope only. In segment $\mathrm{K}$, we gave zero weight to the 
TABLE 2. Parameters of line segment fits.

\begin{tabular}{|c|c|c|c|c|c|}
\hline segment & $\mathrm{n}$ & $\begin{array}{l}\Delta \mathrm{P} \\
\text { (days) }\end{array}$ & $\begin{array}{l}\text { std.dev. } \\
\text { (days) }\end{array}$ & $\begin{array}{l}\text { cycle } \\
\text { number }\end{array}$ & $\begin{array}{l}0-c \\
\text { (days) }\end{array}$ \\
\hline$-\infty-\infty-1$ & $\cdots$ & 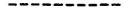 & $-\cdots$ & $-\cdots$ & - \\
\hline A & 67 & $\begin{array}{r}-0.0000722 \\
\pm 54\end{array}$ & 0.0059 & & \\
\hline $\mathrm{A} / \mathrm{B}$ & & & & -3514 & +0.572 \\
\hline B & 37 & $\begin{array}{r}-0.0001604 \\
\pm 56\end{array}$ & 0.0057 & & \\
\hline$B / C$ & & & & -2968 & +0.484 \\
\hline c & 9 & $\begin{array}{r}-0.0002817 \\
\pm \quad 68\end{array}$ & 0.0078 & & \\
\hline$C / D$ & & & & -1945 & +0.196 \\
\hline D & 9 & $\begin{array}{r}-0.0001636 \\
\pm 62\end{array}$ & 0.0041 & & \\
\hline$D / E$ & & & & -1176 & +0.070 \\
\hline $\mathbf{E}$ & 11 & $\begin{array}{r}-0.0000638 \\
\pm 95\end{array}$ & 0.0145 & & \\
\hline$E / F$ & & & & +87 & -0.010 \\
\hline $\mathbf{F}$ & 12 & $\begin{array}{r}+0.0000289 \\
\pm 85\end{array}$ & 0.0052 & & \\
\hline$F / G$ & & & & +729 & +0.008 \\
\hline G & 5 & $\begin{array}{r}+0.0001574 \\
\pm \quad 292\end{array}$ & 0.0191 & & \\
\hline $\mathrm{G} / \mathrm{H}$ & & & & +1161 & +0.076 \\
\hline H & 3 & $\begin{array}{r}+0.0001331 \\
\pm 111\end{array}$ & 0.0019 & & \\
\hline $\mathrm{H} / \mathrm{I}$ & & & & +1601 & +0.135 \\
\hline I & 3 & $\begin{array}{r}+0.0001904 \\
\pm 136\end{array}$ & 0.0022 & & \\
\hline$I / J$ & & & & +2046 & +0.220 \\
\hline $\mathrm{J}$ & 40 & $\begin{array}{r}+0.0000999 \\
\pm 66\end{array}$ & 0.0074 & & \\
\hline $\mathrm{J} / \mathrm{K}$ & & & & +2773 & +0.292 \\
\hline $\mathrm{K}$ & 18 & $\begin{array}{r}+0.0000121 \\
\pm 78\end{array}$ & 0.0074 & & \\
\hline
\end{tabular}

one visual timing at $E=+3015$. Otherwise, all points within each segment were included and given equal weight.

Residuals from the 11 straight-line-segment fits are listed in the third column of Table 1 , as $\mathrm{O}-\mathrm{C}^{\prime}$, and plotted in Fig. 2 , including those points excluded from the least squares solutions.

\section{DISCUSSION OF TIMING ERRORS}

Most papers on period changes in eclipsing binaries underdo critical consideration of the observational errors in the timing data. This oversight has been responsible for instances where the reality of, or nature of, deduced period variations has been doubted. We want, therefore, to provide a frank discussion of the errors in this case, even though the heterogeneity of the data makes that a difficult task.

For the ten nonphotoelectric times with uncertainties specified in Table 1 (Ref. nos. 3, 14, 15, 18) the residuals are generally consistent with those uncertainties. All of the photoelectric timings have residuals between 0.0000 and $\pm 0 \mathrm{~d} 0016$, except for the two of Olson (1982), which Olson himself said were "less reliable." Though very small indeed, most of them are larger than the uncertainties quoted in the original sources, by a factor 2 or 3 on the average. We know of only one paper (Mallama 1974) which provides estimates of errors expected for visual eclipse timings, useful when

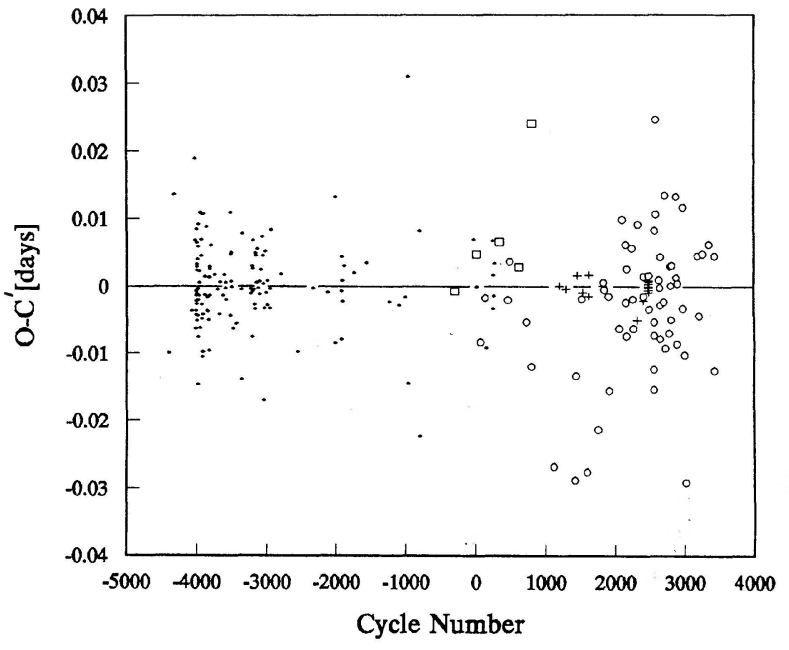

FIG. 2. Residuals from the 11 straight-line-segment fits in table 2. Dots are from Slovochotova (1954) and the two added from Parenago (1931). Squares are photographic timings, including the spectroscopic timing by Struve (1945). Plusses are photoelectric timings. Open circles are timings from visual estimates. For some segments, for example, $\mathrm{H}$ and $\mathrm{I}$, in the range $+1161<E<+2046$, only the photoelectric times were used for the fit by least squares, the others being given zero weight.

explicit error estimates are not published. We find that the average residual of all timings made by the method of visual estimates was quite a bit larger than one would have expected from the Mallama's nomogram. Judging from the primary eclipse depth and average rate of light loss in the visual bandpass, Mallama's two input parameters, visual eclipse timings of SW Cyg should have errors of about \pm 0.002 . The average residual was \pm 0.005 for timings by the

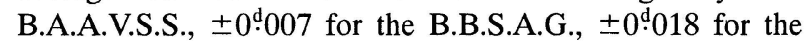
A.A.V.S.O. (Baldwin 1973), and similar for the large number of early (mostly visual) timings listed by Slovochotova (1954): about \pm 0 d 02 for those she had given half weight and about \pm 0.004 for those with weight 1,2 , or 3 . So it seems that the Mallama nomogram underestimates the true uncertainties in this case of SW Cyg, but we can suggest why. Unlike the 15 stars Mallama used in his study, SW Cyg has a long orbital period, too long for a complete eclipse (first to fourth contact) to be observed in a single night. There is, however, no published study comparable to Mallama's which would be applicable to the long-period eclipsing binaries like SW Cyg.

Those instances where the residuals are greater than the expected uncertainty, especially the photoelectric timings, might be accounted for in large part, by light curve asymmetry. Walter (1971) had found the upper half of the descending branch fainter by almost $0 .{ }^{\mathrm{M}} 05$, most probably due to absorption of the A2 V star's light by the gas stream. Hall \& Garrison (1972) and Hall et al. (1979) noted other asymmetries around the internal contacts and discussed them in terms of excess light from circumstellar material around the $\mathrm{A} 2 \mathrm{~V}$ star, though these effects were sizable only in the B and U bandpasses. More on light curve asymmetry in SW Cyg was said by Olson (1982). If one eclipse branch is brighter or fainter than the other by $\Delta m$, then a time of eclipse midpoint 


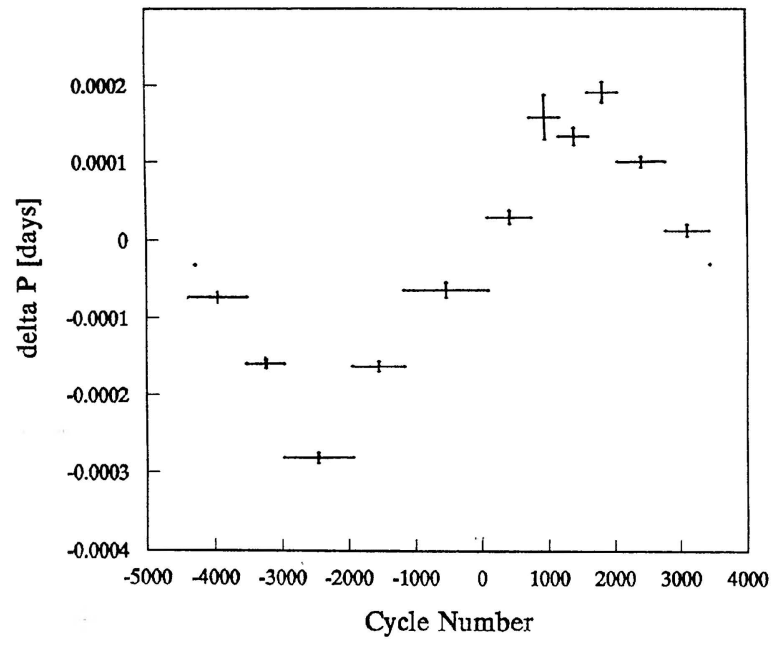

Fig. 3. Periods for the 11 segments, taken from Table 2. $\Delta P$ is difference from the period in Eq. (1). The two dots are short extrapolations to $\Delta P$ $=-0$ d 00003 , used to estimate the cycle length at $96 \mathrm{yr}$.

derived by the method of bisecting chords or an equivalent method, without knowledge of, or proper correction for, the asymmetry, would be in error by

$$
\Delta t=\frac{1}{2} \frac{\Delta m}{d m / d t},
$$

where $d m / d t$ is the average rate of light loss during eclipse, about $0.5 \mathrm{mag} / \mathrm{h}$ for SW Cyg. For $\Delta m=0 .{ }^{\mathrm{M}} 05$, one would get an error in the eclipse timing of \pm 0 d 002 .

Although we have used the method of straight-line segments to fit the 97 yr-long O-C curve of SW Cyg, and have achieved a statistically satisfactory representation, we do not wish to assert that the orbital period in SW Cyg has actually undergone a sequence of nearly instantaneous changes while remaining strictly constant during the intervals between. We prefer to view the straight-line segment approach as a numerical approximation adequate for describing this particular data set. Having said this, we admit that some degree of stepwise change in the period, as opposed to perfectly continuous change, might be occurring. One of the best discussions of this point, in connection with SW Cyg in particular and other eclpsing binaries in general, is the one by FrieboesConde \& Herczeg (1973, Fig. 12). The definitive answer to this question will require more sophisticated analysis of the data and perhaps more data of uniformly higher quality, i.e., good photoelectric timings, properly corrected for asymmetry effects, great in number, pursued for several more decades, with no significant gaps in time. The magnetic cycle model itself, referred to in Sec. 1, provides no guidance. Too little is known about such cycles even to suggest what behavior is to be expected, stepwise or continuous.

\section{THE CYCLE LENGTH}

Figure 3 is a plot of the periods given in Table 2 for the 11 segments of the $\mathrm{O}-\mathrm{C}$ curve. The minimum period occurred in 1918.9, if we take the middle of segment $C$ as the epoch, and the maximum period occurred in 1972.5, the middle of the I segment. If we suppose that the period varies symmetrically in time, then the full cycle length would be $107 \mathrm{yr}$. This supposition may be a poor one, however, because the one magnetic cycle which has been observed most extensively, the solar cycle, is not symmetrical in time. The "butterfly diagram" shows the rise to sunspot maximum faster than the decline to minimum.

Fortunately, only very short extrapolations at both ends of the curve in Fig. 3 yield equivalent values of the period $(\Delta P=-0 \stackrel{0}{d} 00003)$ at $E=-4290$ and $E=+3430$. That would translate to a full cycle length of $96 \mathrm{yr}$. This is probably a more reliable estimate of the length of the cycle we believe is operating in the K-type subgiant of SW Cyg.

Frieboes-Conde \& Herczeg (1973) had already concluded that interpretation of the $\mathrm{O}-\mathrm{C}$ curve as a consequence of the time-delay effect, as SW Cyg orbits around the center of mass between itself and a third body, is "out of the question." If the O-C curve is imagined as a sinusoid, it has a full amplitude of about $0 \div 45$, corresponding to a projected semimajor axis (for the orbit of SW Cyg around the center of mass) of about $40 \mathrm{AU}$. With a period of $96 \mathrm{yr}$, an inclination of $90^{\circ}$, and a total mass of $1.69 \mathscr{C}_{\odot}$ for SW Cyg (Van Hamme \& Wilson 1990, Table VI), one gets $10 \mathscr{M b}_{\odot}$ for the mass of the third body. All other estimates for the mass of SW Cyg appearing in the literature are larger and, with a larger total mass, one gets an even more massive third body. With the 4.0 or $4.6 \mathscr{L}_{\odot}$ suggested by Hall (1974), the largest estimates we can find, one gets 13 or $14 \mathscr{A b}_{\odot}$. And with inclinations less than $90^{\circ}$, one also gets a more massive third body. If, for example, $i=60^{\circ}$ and the masses of Van Hamme and Wilson are retained, one gets $15 \mathscr{M}_{\odot}$. SW Cyg has no visual companion more luminous than itself at the appropriate angular distance in the sky. The faint $\left(V=13^{M}\right.$ or $\left.14^{M}\right)$ companion at $\rho=20^{\prime \prime}$ (Hall \& Garrison 1972) must be at least 2500 AU away from SW Cyg and hence not a candidate. Therefore, such a massive third body is indeed "out of the question," unless that body is an isolated stellar black hole, not just a neutron star (which cannot be more massive than $\left.3 \mathscr{M}_{\odot}\right)$, but actually a black hole.

With its deep convection and rapid rotation, the K-type subgiant in SW Cyg lies well within the domain of "activedynamo stars," those apt to manifest pronounced magnetic phenomena. According to Hall (1991a), the active dynamo stars are those with a Rossby number (ratio of rotation period to convective turnover time) less than $2 / 3$. With its rotation period known ( $4 \cdot 573)$ and its convective turnover time derivable (Hall 1991a) from its temperature (spectral type) and radius (luminosity class), its Rossby number must be around 0.05 , smaller than $2 / 3$ by a wide margin.

In his study of the occurrence of magnetic cycles in stars of various different types, Hall (1990) had found cycle lengths ranging from single-digit years up to almost $175 \mathrm{yr}$, with a median of about 30 yr. Cycle lengths found in the subset of Algol-type binaries (in which one component is convective) ranged from 7 to $109 \mathrm{yr}$, with a median of $50 \mathrm{yr}$ (Hall 1989). Algol itself has a $32 \mathrm{yr}$ cycle (Soderhjelm 1980). The 96 yr cycle we have found in SW Cyg is, in this context, rather long, but still not the longest one of its sort 
ever seen. The size of the period variation, longer than and shorter than the long-term mean of 4.57294 , is $\Delta P / P= \pm 5$ $\times 10^{-5}$, nicely typical of such period changes in similar Algol-type binaries.
We are grateful to Weaver S. Barksdale for a gift, to Dyer Observatory, which has made publication of this paper possible, and to Piotr Flin for help in locating several obscure references to times of minimum which proved to be useful.

\section{REFERENCES}

Applegate, J. H. 1992, ApJ, 385, 621

Baldwin, M. E. 1973, I.B.V.S. no. 795

Blazko, S. 1925, Beobachtungs Zirkular der Astron. Nach., 7, 35

Chaubey, U.S. 1980, Ap\&SS, 67, 129

Flin, P. 1983, M.V.S. 9, 89

Frieboes-Conde, H., \& Herczeg, T. 1973, A\&AS, 12, 1

Hall, D. S. 1968, IBVS, No. 281

Hall, D. S. 1974, AcA, 24, 215

Hall, D. S. 1989, IAU Colloquium No. 107, 219

Hall, D. S. 1990 , in Active Close Binaries, edited by C. Ibanoglu (Kluwer,

Dordrecht), p. 95

Hall, D. S. 1991a, IAU Colloquium No. 130, 353

Hall, D. S. 1991b, ApJ, 380, L85

Hall, D. S., \& Garrison, L. M. 1972, PASP, 84, 552

Hall, D. S., Quarles, T., Roberts, J., \& Whelan, J.A.J. 1979, AcA, 29, 653

Isles, J. E. 1988, JBAA, 98, 200

Kordylewski, K. 1963, IBVS No. 35

Lange, G. A. 1959a, Astr. Circ. No. 200, 14

Lange, G. A. 1959b, Astr. Circ. No. 201, 13

Lange, G. A. 1960, Astr. Circ. No. 209, 23
Mallama, A. D. 1974, J.A.A.V.S.O., 3, 11

Mallama, A. D. 1980, ApJS, 44, 241

Olson, E. C. 1981 , IBVS No. 1938

Olson, E. C. 1982 , ApJ, 259, 702

Parenago, P. P. 1931, Russian AJ, 8, 229

Pickering, E. C. 1899, ApJ, 10, 126

Rafert, J. B. 1982, PASP, 94, 485

Schneller, H. 1963, A.N., 287, 183

Scholz, S. 1964, Harthaer Beobachtungs Zirkular No. 28

Slovochotova, N. P. 1954, P.Z., 10, 21

Soderhjelm, S. 1980, A\&A, 89, 100

Struve, O. 1946, ApJ, 104, 253

Szczepanowska, A. 1956, AcA, 6, 144

Tout, C. A., \& Hall, D. S. 1991, MNRAS, 253, 9

Van Hamme, W., \& Wilson, R. E. 1990, AJ, 100, 1981

Walter, K. 1971, A\&A, 13, 249

Whitney, B. S. 1959, AJ, 64, 258

Wilson, R. E., \& Mukherjee, J. 1988, AJ, 96, 747

Wood, D. B., \& Forbes, J. E. 1963, AJ, 68, 257 